\title{
Zero Ischemia Partial Nephrectomy in Pregnancy
}

\author{
Omer Vefik Ozozan1, Abidin Egemen Isgoren² \\ ${ }^{1}$ Department of General Surgery, Istinye University Bahcesehir Hospital, Istanbul, Turkey \\ 2Department of Urology, Memorial Antalya Hospital, Antalya, Turkey
}

\begin{abstract}
Renal cell carcinoma (RCC) represents $2-3 \%$ of all cancers. Although RCC is rare, it is the most common urologic malignancy reported in pregnancy. Modality and timing of treatment during pregnancy represent a dilemma. A 37-year pregnant woman at $12^{\text {th }}$ gestational week was referred by the obstetrician. MRI showed exophytic, heterogeneous renal mass of $50 \times 48 \times 45 \mathrm{~mm}$, located in the lower pole of right kidney, which was interpreted as RCC. The patient underwent retroperitoneal open partial nephrectomy (PN). Operation time was 47 minutes with zero ischemia time. Obstetrics consultation confirmed survival of fetus after surgery. The patient was discharged on the third day, uneventfully. Histopathological examination revealed stage $1 \mathrm{~b}$ clear-cell renal carcinoma, Furhman grade 2 with maximum diameter of $5 \mathrm{~cm}$, no invasion of capsule, negative margine and without necrosis. Open techniques are better in pregnant patients with renal tumors because of short surgical and ischemia time and minimal bleeding.
\end{abstract}

Key Words: Renal cell carcinoma, Zero ischemia, Partial nephrectomy.

How to cite this article: Ozozan OV, Isgoren AE. Zero ischemia partial nephrectomy in pregnancy. J Coll Physicians Surg Pak 2020; 30(1):88-89.

\section{INTRODUCTION}

Renal cell carcinoma (RCC) represents $2-3 \%$ of all cancers. Although RCC is rare in women of childbearing age, it is the most common urologic malignancy reported in pregnancy with a rate of $50 \%$, followed by bladder cancer and adrenal tumors, especially pheochromocytoma. With the use of routine antepartum ultrasound, there is an increase in the incidental detection of RCC during pregnancy. 1

Treatment of RCC during pregnancy represents a dilemma. Surgical decision is individualised for each case. Although new targeted agents have been developed, the effect of these on fetal and maternal outcomes is poorly studied.2 On the other hand, based on current knowledge, localised RCCs are better managed by partial nephrectomy (PN) rather than radical nephrectomy $(\mathrm{RN})$.

Multiple retrospective series, as well as one prospective randomised controlled trial (RCT) including patients with organ-confined RCC of limited size (pT1), have demonstrated a comparable cancer specific survival (CSS) for PN vs. RN. ${ }^{3}$

Timing of surgery is crucial for RCC in pregnant women. Delaying the surgery to post-delivery may lead to fatal consequences for mother and fetus. ${ }^{4}$

Correspondence to: Dr. Omer Vefik Ozozan, Department of Surgery, Istinye University Bahcesehir Hospital, Istanbul, Turkey

E-mail: omerozozan2002@yahoo.com

Received: August 27, 2019; Revised: October 25, 2019; Accepted: October 25, 2019
Although the most of the PN cases are being performed by using minimal invasive techniques, fetus is vulnerable bacause of prolonged surgical time and blood loss. Therefore, we advocate that pregnant women with renal tumors are better to be treated by open techniques, because of short surgical and ischemia time and minimal blood loss.

To best of our knowledge, we hereby report the first zero ischemia $\mathrm{PN}$ case during pregnancy.

\section{CASE REPORT}

A 37-year pregnant woman, G3, P2, L2, at her $12^{\text {th }}$ gestational week (GW), was referred to our clinic by an obstetrician, who had diagnosed a mass in her right kidney by ultrasonography at $10^{\text {th }} \mathrm{GW}$ with right flank and loin pain as RCC. Renal vein invasion and lymph node involvement was not detected.

She had no previous history of hypertension, gestational diabetes mellitus, renal disease, or pre-eclampsia. She did not have any family history of RCC and no genetic abnormalities identified earlier. No pathological findings in ultrasound was performed one year ago.

Physical examination of the patient was unremarkable. Her chest radiograph, and blood tests were normal. After further obstetrics consultation, possible treatment alternatives were discussed with the patient; and open retroperitoneal PN was decided.

Partial nephrectomy decision was made because of tumor location, tumor size, and low PADUA score. The patient underwent retroperitoneal $P N$ at $14^{\text {th }} \mathrm{GW}$ under general anaesthesia by flank incision. After opening 
Gerota's fascia and localising the kidney, the substantially exophytic tumor was detected. The renal pedicle was identified and released, isolated the main renal artery and vein. Tumor resection was completed allowing a safety margin of healthy renal parenchyma. The resection bed was carefully inspected to avoid opening of the urinary passage; and hemostasis was done rapidly with running stitches of $4 / 0$ absorbable suture. Operation time was 47 minutes with zero ischemia time. Estimated blood loss was $50 \mathrm{ml}$. No blood tranfusion was required. No complication occurred during or after the surgery.

Obstetric consultation confirmed the survival of the fetus just after the operation. Postoperative hemoglobin was $10.3 \mathrm{~g} / \mathrm{dL}$ and hematocrit $29.9 \%$. Leukocytes and platelet count were all within normal limits. BUN and creatinine were $4 \mathrm{mg} / \mathrm{dL}$ and $0.44 \mathrm{mg} / \mathrm{dL}$, respectively. The patient was discharged from the hospital on the third day after surgery, uneventfully.

Histopathological examination revealed a stage $1 \mathrm{~b}$ clearcell renal carcinoma, Furhman grade 2 with maximum diameter of $5 \mathrm{~cm}$, no invasion of the renal capsule, negative surgical margine and without necrosis. No recurrence at 1-year follow-up was observed.

\section{DISCUSSION}

$\mathrm{RCC}$ is a very rare clinical situation during pregnancy; approximately 102 cases have been reported in literature. Presentation of RCC in pregnant women is often with pain and hematuria; while classical triad of pain, palpable mass, and hematuria is present in $26 \%$ of cases. ${ }^{1}$ Symptoms such as pain and hypertension may mimic symptoms of common pregnancy-related complaints. The presence of loin or abdominal pain, hematuria or hypertension should be kept in mind in diagnostic evaluation with an abdominal ultrasound to allow early diagnosis of such malignancies.

GW and the relatively slow doubling time of RCC play an important role in the timing for surgery. ${ }^{5}$ Patients diagnosed during the first or the third trimester could be good candidates for surgery. However, for patients diagnosed during the second trimester, decision making is not clear. ${ }^{6}$ During the first trimester, there is increased risk to the life of the fetus; whereas, surgical treatment in the second and third trimester is considered to be reasonable and safe after careful consideration of the maternal and fetal health. During second trimester, surgical manipulations may induce uterine contractions causing spontaneous abortion; also hypotension during surgery caused by blood loss can lead to fetal hypoxia.

Although the recommended treatment reported in literature for RCC in pregnancy continues to be RN, nephron-sparing surgery and laparoscopic approach can be alternative options in selected patients. ${ }^{7}$

\section{CONFLICT OF INTEREST:}

Authors declared no conflict of interest.

\section{AUTHORS' CONTRIBUTION:}

ÖVÖ: Drafting the work,revising critically,final approval of the version to be published

AEl; Substantial contributions to the conception,analysis or interpretetion of data for the work.

\section{REFERENCES}

1. Boussios S, Pavlidis N. Renal cell carcinoma in pregnancy: A rare coexistence. Clin Transl Oncol 2014; 16:122-7.

2. Escudier B, Porta C, Schmidinger M, Algaba F, Patard JJ, Khoo V, et al. Renal cell carcinoma: ESMO clinical practice guidelines for diagnosis, treatment and follow-up. Ann Oncol 2014; 25(suppl 3):iii49-56.

3. Van Poppel H, Da Pozzo L, Albrecht W, Matveev V, Bono A, Borkowski $A$, et al. A prospective, randomised EORTC intergroup phase 3 study comparing the oncologic outcome of elective nephron-sparing surgery and radical nephrectomy for low-stage renal cell carcinoma. Eur Urol 2011; 59:543-52.

4. Renshaw AA, Granter SR, Fletcher JA, Kozakewich HP, Corless CL, Perez-Atayde AR. Renal cell carcinomas in children and young adults: Increased incidence of papillary architecture and unique subtypes. Am J Surg Pathol 1999; 23: 795-802.

5. Tiang KW, Ng KL, Vega-Vega A, Wood S. Rapidly enlarging renal tumor during pregnancy: Diagnostic and management dilemma. J Kidney Cancer VHL 2014; 1:12-6.

6. Khochikar MV. Management of urological cancers during pregnancy. Nat Rev Urol 2010; 7:195-205.

7. Ljungberg $\mathrm{B}$, Albiges $\mathrm{L}$, Abu-Ghanem $\mathrm{Y}$, Bensalah $\mathrm{K}$, Dabestani S, Fernández-Pello $\mathrm{S}$, et al. European Association of Urology Guidelines on Renal Cell Carcinoma: The 2019 Update. Eur Urol 2019; 75:799-810. 